\title{
LATENT FINGERPRINT AND VEIN MATCHING USING RIDGE FEATURE IDENTIFICATION
}

\author{
Rajeswari.P ${ }^{1}$, Sathya. $\mathbf{M}^{2}$, Joy Kinshy. $\mathbf{P}^{3}$ \\ ${ }^{1} P G$ Scholar, Computer Science and Engineering, Knowledge Institute of Technology, Salem, TN, India \\ ${ }^{2}$ Assistant Professor, Computer Science and Engineering, Knowledge Institute of Technology, Salem, TN, India \\ ${ }^{3}$ PG Scholar, Computer Science and Engineering, Knowledge Institute of Technology, Salem, TN, India
}

\begin{abstract}
Fingerprint is an impression of friction ridges of the fingers. It has getting better either by enrollment or by the impressions lifted from crime scenes. Latent prints are partial prints, invisible and it had been get from accidental impressions of crime scenes. Minutiae matches had been defined as the points which are marked with 3 different regions like start, end and intersection ridges of the fingerprints. For an authentication system, full-to-full matching fingerprint are efficient. But it is not efficient in latent-to-full matching fingerprints. In our proposed system, latent-to-full matching fingerprint must be efficient with the distance had been calculated with in the minutiae points by using $8 \times 8$ Gabor filter, using this spatial frequencies had been calculated. In this case, the spoof attacks had been occurred. To reduce these attacks, we propose an algorithm, Ridge Feature Identification and it may combine the work of taking distance minutiae calculation and the finger-vein matching to become the system more efficient and provides the liveliness in the authentication system.
\end{abstract}

Keywords: Fingerprint, Latent prints, Ridge Feature Identification, Minutiae matches, Finger-vein.

\section{INTRODUCTION}

Now a days, Biometrics maps to a new civilian applications of commercial use. Automatic Fingerprint Identification System (AFIS) appeared as an essential tool for all the biometrics and the law enforcement agencies [1].The demand of the secured biometric system, now lead to the growth in the features and individualities for the responsibility of quite complicated fingerprint and finger-vein present in the fingertips of the fingerprint. This important technology is widely used in areaaccess control, Pc login, and e-commerce. These all biometrics are attracted towards this technology, because of password theft, loss and release of user's memory [10].

In biometrics system, the security and convenience of the system are important [10].The system requires high response times and fast accuracy. The biometrics includes the patterns based on fingerprint, finger-vein, facial features, the iris, and the voice, hand geometry. However, some of the features are susceptible to forgery, so if it is used in combination the system will be efficient. The fingerprint and finger-vein produces high confidentiality over other measures.

\subsection{Fingerprint}

In general, not even twin sisters or twin brothers may not have the same fingerprint. They may be varying with respect to their genetic arrangements of cells and tissues. Fingerprint may consist of friction ridges and it is a combination of ridges(black line) and valleys(space between the two
ridges).In fingerprint,level-2 fingerprint features has been executed with high performance and individuality in the fingerprints and it may produce the imperfections randomly in the case of friction ridges and they are known as the minutiae. These type of fingerprint acquisition typically utilizes the fingerprint resolution must higher than 410dpI.Although,[2]level-1 features may be utilized by Automated Fingerprint resolution, and this may produce the pattern type and ridge flow features present in our fingers and it may be got from a low-resolution images, and this features are used only for fingerprint classification.

Fingerprint [6] images are of three types. (i) rolled, (ii)plain and (iii)latent. Although rolled and plain fingerprint images are used for forensic applications, whereas rolled print may be used for government applications and commercial one. This may have the friction ridge impressions of full nail (end-toend) for the purpose of registry.

But in plain fingerprint, once the finger is pressed in a flat surface and not be rolled, both this plain and rolled prints are taken by the live scan machine or inked impressions on paper. Latent fingerprint is defined as the partial print or injured print. It defines that the impressions we get it in a damaged conditions or partial prints. But half of the fingerprint is there to identify a system.

In identification system, the enrolled fingerprint is stored at an initial stage and the verifying fingerprint is the print for the 
authentication purpose, we have to verify among the enrolled prints. If the fingerprint is plain or rolled, it is easy to verify the system in AFIS. But if it is latent, the identification system is not successful and it is failed. So [1], the fingerprint examiners of manual identifiers, use the procedure ACE-V in the latent fingerprint Identification System. The ACE-V procedure includes (Analysis, Comparisons, Evaluations and Verifications).

Step 1: First, Analysis the image whether rolled, plain or latent [3]. It may verify that the sufficient ridge information is available and mark the features along with associated quality information done by human experts.

Step 2: Compared to the original image in the database (includes the level 1, level 2 and level 3).

Step 3: Evaluate and classify the fingerprint pair as individualization (match or non-match).

Step 4: By verifying and check the result by another examiner, to verify the identity is correct or not.

The analysis and identifications seems to be critical issues. The errors may be done by the human examiners like erroneous exclusion and erroneous individualization[3].The minutiae features includes ridges intersection, ridge bifurcation, ridge starting, ridge ending provides the marking of those particular region and performs the action by taking the matching score between the enrolled and verifying fingerprints and that can be performed by baseline matching algorithm. Focus on this method includes the local minutiae matching; global minutiae matching and matching score computation are efficient in this method.

In many aspects, Feature extraction is a major process. In this feature extraction, the features are defined in [3], CDEFFS document and use the term consistent here (1) Reference points includes type, location and directions. (2) Ridge flow map (3) ridge wavelength map (4) Ridge quality map. By these features, the feature extraction is efficient over it. This may be generated based on the validated ridges.

In relevant aspects, Anil k.Jain et.al [1] using Hough transform is to evaluate the straight lines present in a fingerprint images. Based on these observations, edge detection is the reprocessing procedure in this method. The process includes (1) get an imperfect image as input (2) extent the identity by using arbitrary shapes. Besides, on latent fingerprint enhancement [6] can be done by providing Gabor filter for enhancement purpose. Here, it has two parameters, (1) local ridge orientation and (2) frequency generation. According to this method, Gabor filtering can connect the broken ridges and separate the joined ridges. Here, in this method, the true ridges are weakened and spurious ridges are strengthening after this Gabor filtering.

Focus on this previous method, Image segmentation is an important method in fingerprint image. This method may segment the image into a set a pixels and individual pixel may consist of useful information about the minutiae. Now a day, boundary detection using segmentation is efficient and label formation is the enhancing technique.

\subsection{Finger-Vein}

Finger-vein recognition is a technique to provide liveliness in the sensor-level spoof attacks[2].In this recognition technique, it may provide the finger response to electrical signals, temperature and electrocardiograph signals in the finger, time varying perspiration from fingertips and percentage of generated oxygen saturated hemoglobin in the blood.

Now a day, it is almost used in biometric and forensics, the vein images are acquired from the near-infrared-based or thermal-infrared-based optical imaging technique. This optical imaging technique is economically feasible and sensitive to skin as a deeper tissue features.

The finger-vein patterns can be extracted by placing the finger in between the camera and infrared light source [10].The infrared light is passed through the hands in the backside of finger and the hemoglobin absorbs the light and produces the patterns of veins in the palm side of the hand as shadows. It consists of vein patterns and irregular shading and noise.

Almost, in a single image, the vein patterns are not clear and it consists of thickness of finger bones and muscles. So, simultaneous extraction of finger-vein patterns is efficient and useful. Personal Identification using finger vein patterns has received a lot of interest in research area of forensic and civilian application [2] [10].

Miura and Nagasaka et al. [10] using a repeated line tracking algorithm to further improve the performance of vein identification. A detailed evaluation of this method may evaluate the robustness in the extraction of finger vein has been improved randomly by using the local maximum curvature.

In [2], improves the performance metrics by using the fingervein and low-resolution fingerprint images combines these in a new score level combination. In this finger-vein identification system, extract the shape and improves the accuracy in this system. By using this [10], this system may not achieves the high individuality in very large population.

\section{RELATED WORK}

In this section, we reviewed the related work in two areas. One with fingerprint and the other work with finger-vein. Mostly, all the algorithms work with the help of the minutiae points present in the fingerprint. In this method [1], feature extraction is the initial process and it extracts the minutiae and orientation field from both the latent print and rolled print. With the use of the gradient based method, local minutiae 
descriptors are built from the minutiae and orientation field is also reconstructed.

\section{Local minutiae descriptors}

In these descriptors, the minutiae points are coordinated and formed as the cylinder like shape with varying size; it is described as minutiae cylinder code (MCC) by the density of neighboring minutiae in a particular direction.

\section{Orientation Field Reconstruction}

Gradient based method used in this method and the orientation field is also reliable with good images and reconstructed only under the convex hull of minutiae points.

$$
S m=\frac{1}{N} \sum_{i-1}^{n} S c(i) S s(i)
$$

Where,

$\mathrm{Sm}=$ matching minutiae score around two fingerprints. $S c(i)=$ the $i$ Th pair in matched minutiae cylinder code.

$$
S S(i)=1-\left(\frac{d s(i)}{2 t s}\right)
$$

Where,

$d s(i)=$ spatial distance.

$N=$ number of minutiae in the latent.

Feng et al. [12] extract the fingerprint features and the features had been marked in the particular fingerprint. Based on this extracted fingerprint, the orientation field is estimated and the curvature field also constructed from this features. The pyramid formation of fingerprint is made and extracts the singular points at lower and higher levels.

Almost in filtering large fingerprint database for latent matching [6], the features in fingerprint includes 3 levels of resolutions includes minutiae, pores and ridge shapes. Here [6] described a minutiae-based method and the features includes the feature marking and feature extraction at lost the filtering techniques are enhanced and the matching process starts and identify a particular person in a system.

The ridge lines present in the fingerprint evaluates the orientation and curvature field is computed. The singular point extracts at both high and lowers level locations. The pattern type is also recognized as loop, tented arch, plain arch, whorl etc. This system may propose the multistage filtering in this fingerprint database as follows: singularity filtering and orientation filtering.

Jianjiang Feng [5] described the method of descriptor-based minutiae matching algorithm and combining the information of texture and the minutiae information is performed. The minutiae correspondence problem solved with a simple alignment-based greedy matching algorithm. By this method, the performance rate may be increased correspondingly.

In [13], exposed the system with reduced manual input and improves the matching accuracy. This enhancement algorithm manually marked the ROI and the singular points. The commercial SDK provides the enhancement algorithm in an orientation field estimation algorithm and fits it to the coarse field estimation as a skeleton. This system performs the operation of 'lights-out 'mode in an efficient way.

The proposed method in [2] can acquired simultaneous fingervein and finger surface images are presented. This method illustrates and utilizes more user-friendly and peg-free imaging. The steps in this method include image normalization, segmentation and rotation of the interclass image had been developed.

Anil K.Jain et.al [3] used local minutiae matching, global minutiae matching and matching score computation. This [3] may clearly demonstrates the features of the minutiae points and the lines present. Naoto Miura et.al [10] used the repeated line tracking method, to detect the patterns of veins and removed by background by blacked out regions. The pixel marked as a current tracking point is recorded as a matrix named as "locus space". The locus space values have the high or frequency.

In[13],includes four steps to enhance the algorithm,1)manual markup of ROI and singular points 2)STFT 3)Orientation field estimation 4)Gabor filters.

\section{METHODS AND DATA ANALYSIS}

\subsection{User Interface Design}

The objective of user interface design is to make the user's interaction as simple and more efficient as possible, in terms of completing user goals is often referred as user-centered design. Good customer boundary design accelerates concluding the task at hand without drawing fired attention to it. Graphic design can be exploited to upkeep its usability and visibility. The design process must balance with the technical functionality and visual elements are used to create a system that is not only operational but also usable and adaptable to changing user needs. 


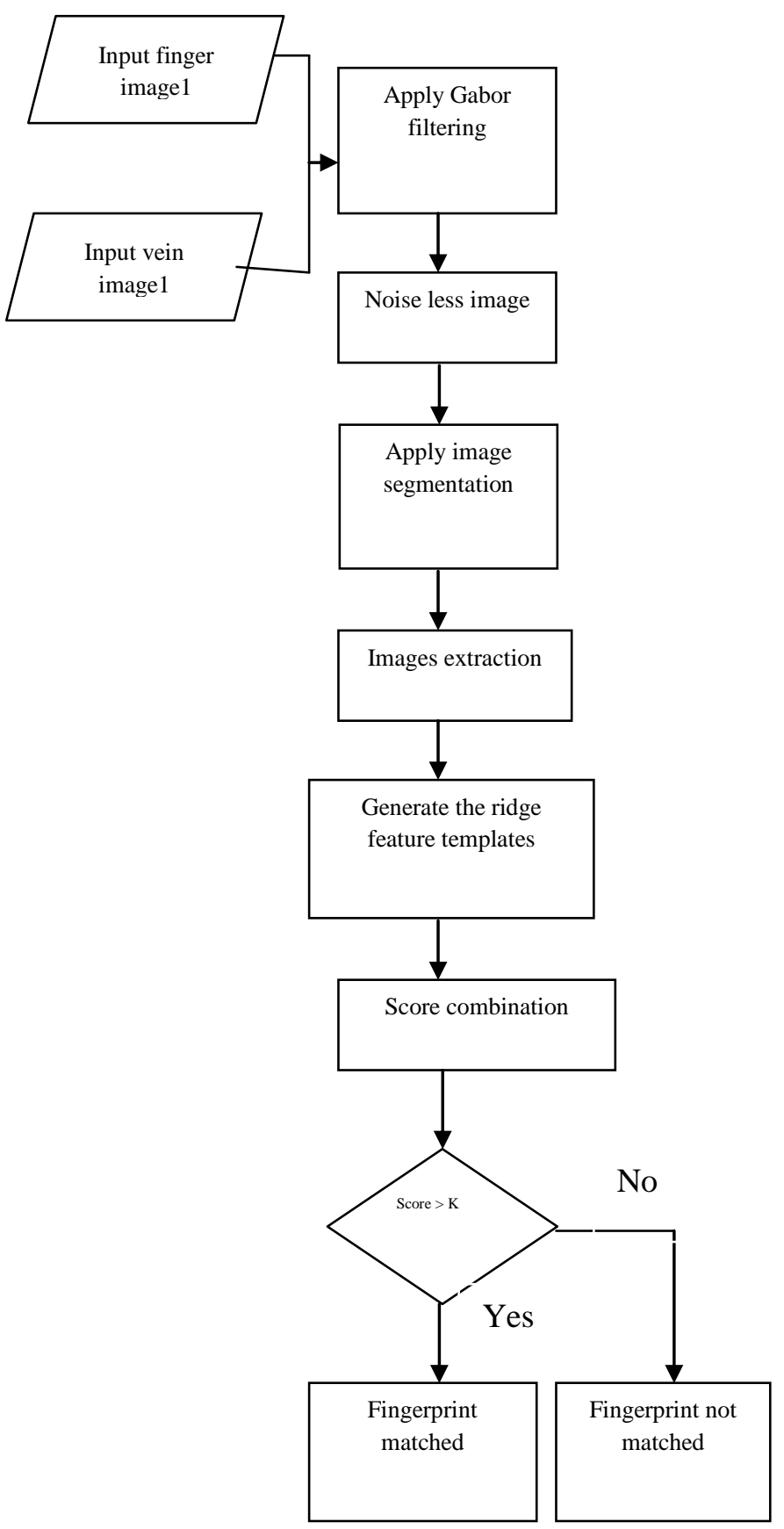

Fig-1 System Flow Diagram for the proposed system

\subsection{NBIS Segmentation Algorithm}

This algorithm segment the original fingerprint image without line removal process. In this algorithm, the background is indicated by the blacked out region. The fig1 demonstrates clearly that the detected fingerprint regions from the proposed segmentation algorithm are much smaller and more accurate than those of the previous method. The ability of the proposed algorithm to reduce the searchable fingerprint area while improving accuracy can be visualized.
In this segmentation process fig.2, take a wide database with rolled fingerprint, the features of the full fingerprint is stored in the NBIS database. At a verification process, a latent of the individual is taken and it had been matched with the database by preceding the first process segmentation. It is a method of splitting a digital image into multiple segments and this leads to simply and change the representation of an image into more useful and easy to analyze .This process had been segmented by using label for every pixels and the pixels with the same label share certain characteristics.

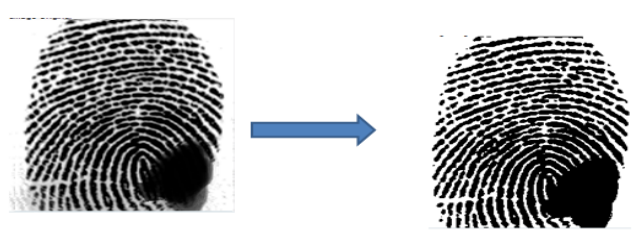

Fig- 2 Segmenting a Latent Image

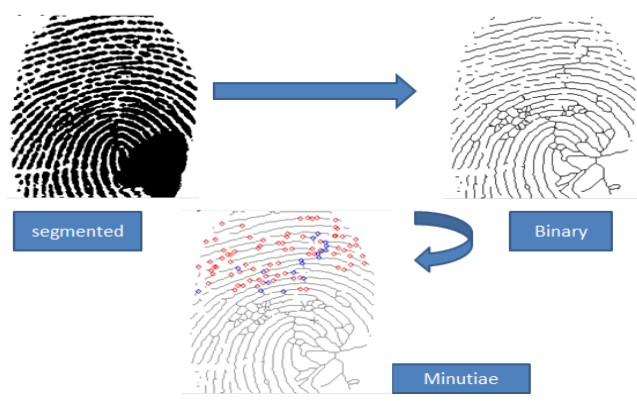

Fig.-3 Binarizing and Minutiae Point Classification

\subsection{Latent Matching Approach}

Prearranged a latent fingerprint and a rolled fingerprint, this method extract extra features from both prints, align them in the similar coordinate system, and calculate a match score amongst them.

\subsubsection{Latent Fingerprint Matching}

Enhanced latent matching accuracy has been described by expending features, which are manually noticeable for latent. However, marking extended features (orientation field, ridge skeleton, etc.) in poor quality latent is exact time-consuming and might be only practicable in infrequent cases. However, only a minor portion of latent can be properly known using this approach. The segmented image is extracted and formed the binary image by the process of binarization.

\subsubsection{Feature Extraction}

The planned matching approach uses minutiae and orientation field from both latent and rolled prints. Minutiae are manually marked by latent examiners in the latent, and automatically 
extracted using commercial matchers in the rolled print. Based on minutiae, local minutiae descriptors are constructed and used in the proposed descriptor-based alignment and scoring algorithms.

\subsection{Ridge Feature Process}

Here the process obtains the essential ridges and minutiae information from the fingerprint image. This method can then define ridge coordinates and extract ridge features between the two minutiae points. Here, the ridge count (rc) is calculated by with the number of ridges along the perpendicular axis until the axis lights the ridge attached to the adjacent minutia. The ridge length ( $\mathrm{rl})$ is the distance on the horizontal axis from the joint of the perpendicular and horizontal axis to a minutia. In addition, the process improves the discriminating control of ridge structures, the ridge type (rt) is used as one of the ridge structures in its place of a minutia type. To regulate the ridge type (rt), all minutia is first ordered as an end point or a bifurcation. If a minutia is an finale point, there is only single ridge fitting to the minutia. If a minutia is a branching, there are three ridges joined to the minutiae.

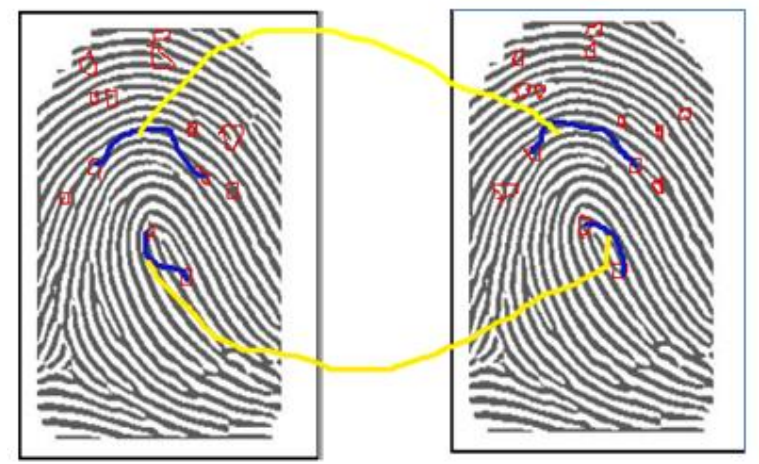

Fig-4 Distance Calculation of Minutiae Points a) red-minutiae points b) blue-distance of minutiae points c) yellow-matching process

\subsection{Fingerprint and Vein Segmentation}

In vein segmentation, the algorithm without the line removal, the background is labeled by the blacked out region. The rushed white outlines were drawn manually and are replicated in the other images to deliver visual orientations for comparison. This algorithm demonstrates clearly that the identified fingerprint regions from the proposed segmentation algorithm are much smaller and more accurate than those of the earlier method. From this method, the capacity of the proposed algorithm to reduce the searchable fingerprint area while improving accuracy can be visualized.

The low contrast images, due to the light scattering outcome, are improved and the fingerprint lines are detached using 2D discrete wavelet filtering. Kernel filtering produces multiple images by rotating the kernel in six dissimilar directions, emphasis into the predictable ways of the vein patterns. The maximum of all images is converted into a binary image. Further development is achieved by a two-level morphological process: a majority filter smoothed the shapes and eliminates some of the misclassified isolated pixels, and a reconstruction procedure removes the remaining misclassified areas.

\subsection{Matching Process}

The complete flow of the proposed fingerprint matching algorithm is as follows:1) Originally match any pair of ridgebased coordinate systems mined from the enrolled fingerprint image and the input fingerprint image using dynamic programming.2) Select the uppermost degree of corresponding ridge-based coordinate pairs.3) For every primarily matched pair, a breadth-first search (BFS) is achieved to detect the matched ridge-based coordinate pairs incrementally.4) Patterned the strength of the matched coordinate pairs using the comparative position and orientation of the minutiae and count the number of matched minutiae.5) Repeat steps 3 or 4 times and then yield the maximum number of matched minutiae.6) Calculate the matching score. If the two image score is matched with other image score this system give the outcome as both are same fingerprint image else both are not same image.

\section{EXPERIMENTAL ANALYSES}

In order to determine the performance enhancement using the proposed schemes, we performed difficult experiments on our collected database. The nonlinear score combination may adjust the matching score by the degrees of consistency between the two matching score as illustrated below:

$$
\wedge=\left(\frac{a+s t}{a+s v}\right)^{\gamma} *(a+s v)^{2}
$$

Where,

$\mathrm{a}=$ positive constant and it is fixed to 1 . $\gamma=$ range of image is selected as $[1,2]$.

$s t=$ matching score from finger texture. $s v=$ matching score from finger-vein.

$\wedge$

${ }_{S}=$ combined score.

Where the equation evaluates that the two matching scores are consistent then the final score is distributed by the vein matching. If it is inconsistent, the matching score provided by the modulated joint probability. In most circumstances, the finger-vein matching is more stable, then the whole matching system is more or less stable.

The model evaluation of proposed system can be measured by considering the environment as follows: 
Table-1 Terms and performance of the system

\begin{tabular}{|l|l|l|l|}
\hline Terms & $\begin{array}{l}\text { Fingerpri } \\
\text { nt }\end{array}$ & $\begin{array}{l}\text { Finger- } \\
\text { vein }\end{array}$ & $\begin{array}{l}\text { Combine } \\
\text { d Results }\end{array}$ \\
\hline Performance & 89 & 88 & 92 \\
\hline Minutiae Points & 69 & 5 & 75 \\
\hline $\begin{array}{l}\text { Distance } \\
\text { Calculation }\end{array}$ & 96 & 7 & 98 \\
\hline Security & 90 & 90 & 99 \\
\hline Authentication & 86 & 86 & 95 \\
\hline
\end{tabular}

The above matrix demonstrates the performance and terms in case of the approximation by using the algorithm level description. First, the performance of the system had been demonstrated and the match score is consistent high. Second, minutiae points had been extracted that shows the combined results with reduced factors. Then, the distance minutiae calculation by using Gabor filter is demonstrated with very high performance. And the security and the authentication is also high is this system of measurements.

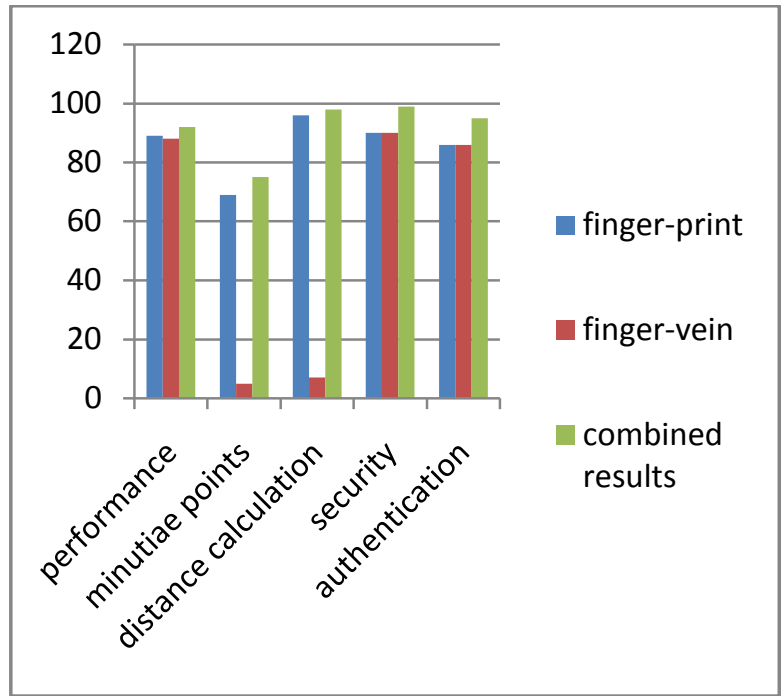

Chart-1 Performances of the Overall System

The overhead chart embodies the whole performance of the latent fingerprint and finger-vein system from the matrix of the Table 1 .

\section{CONCLUSIONS AND FUTURE \\ ENHANCEMENT}

Fingerprint matching algorithm designed for matching latent to rolled/plain fingerprints which is based on a descriptorbased Hough Transform alignment. A comparison between the alignment performance of the proposed algorithm and the well-known Generalized Hough Transform shows the superior performance of the proposed method. Here reported matching results for two different latent fingerprint databases with a large background database of around $32 \mathrm{~K}$ rolled prints. The comparison performance of the proposed matcher with three different state-of-the-art fingerprint matchers. Experimental results show that the proposed algorithm performs better than the three fingerprint matchers used in the study across all image qualities. A score-level fusion of the proposed matcher and one of the commercial matchers shows a further boost in the matching performance. Latent fingerprint used in this descriptor based Hough Transform compares only the minutiae features like ridge start, end and bifurcations. In our work, enhancing this project by taking the distance between the minutiae points and compare with the latent image. Fingervein matching is using in this project to overcome the spoof attacks in this latent fingerprint matching . This may be done by using the Gabor filter in ridge feature identification. This may provide the authentication in an efficient way. In future enhance this system by applying various algorithms with different databases.

\section{REFERENCES}

[1]. A. A. Paulino, J. Feng, and A. K. Jain, "Latent fingerprint matching using descriptor-based Hough transform," in Proc. Int. Joint Conf. Biometrics, pp. 1-7, Oct. 2011.

[2]. Ajay Kumar and Yingbo Zhou, "Human Identification Using Finger Images," IEEE Trans. On Image Processing, vol. 21, no. 4, Apr 2012.

[3]. A. K. Jain and J. Feng, "Latent fingerprint matching," IEEE Trans. Pattern Anal. Mach. Intell., vol. 33, no. 1, pp. 88-100, Jan. 2011.

[4]. D. Maltoni, D. Maio, A. K. Jain, and S. Prabhakar, Handbook of Fingerprint Recognition, 2nd ed. New York: Springer-Verlag, 2009.

[5]. J. Feng, "Combining minutiae descriptors for fingerprint matching," Pattern Recognit., vol. 41, pp. 342-352, 2008.

[6]. J. Feng and A. K. Jain, "Filtering large fingerprint database for latent matching," in Proc. ICPR, Dec. 2008, pp. $1-4$.

[7]. J. Qi, S. Yang, and Y. Wang, "Fingerprint matching combining the global orientation field with minutia," Pattern Recognit. Lett., vol. 26, pp. 2424-2430, 2005.

[8]. J. Gu, J. Zhou, and C. Yang, "Fingerprint recognition by combining global structure and local cues," IEEE Trans. Image Process., vol. 15, no. 7, pp. 1952-1964, Jul. 2006.

[9]. M. Tico and P. Kuosmanen, "Fingerprint matching using and orientation based minutia descriptor," IEEE Trans. Pattern Anal. Mach. Intell., vol. 25, no. 8, pp. 1009-1014, Aug. 2003

[10]. Naoto Miura, Akio Nagasaka, Takafumi Miyatake, "Feature extraction of finger-vein patterns based on repeated line tracking and its application to personal identification" Machine Vision and Applications ,Feb.2004

[11]. R. Cappelli, M. Ferrara, and D. Maltoni, "Minutia cylinder-code: A new representation and matching technique 
for fingerprint recognition," IEEE Trans. Pattern Anal. Mach. Intell., vol. 32, no. 12, pp. 2128-2141, Dec. 2010.

[12]. S. Yoon, J. Feng, and A. K. Jain, "Latent fingerprint enhancement via robust orientation field estimation," in Proc. Int. Joint Conf. Biometrics, New Delhi, India, Oct. 2011, pp. $1-8$.

[13]. S. Yoon, J. Feng, and A. K. Jain, "On latent fingerprint enhancement," in Proc. SPIE, Apr. 2010, vol. 7667, no. 766707.

\section{BIOGRAPHIES}

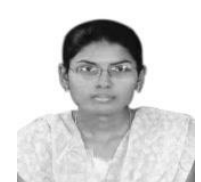

RAJESWARI.P is a student pursuing PG Degree in Computer Science and Engineering at knowledge institute of technology, Salem. Research Interest includes Image Processing contact:rajiprd2@gmail.com Information Security.

SATHYA.M is an Assistant Professor in Knowledge Institute of echnology,Salem.Research Interest includes Image Processing and Web Mining.

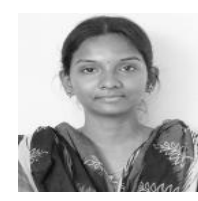

JOY KINSHY.P is a student pursuing PG Degree in Computer Science and Engineering at knowledge institute of technology, Salem. Research Interest includes Image Processing and Mobile Computing. 